\title{
Pumping through a Luttinger liquid ring threaded by a time-varying magnetic field
}

\author{
Enrico Perfetto \\ Dipartimento di Fisica, Universitá di Roma Tor Vergata, Via della Ricerca Scientifica 1, I-00133 Rome, Italy \\ Michele Cini \\ Dipartimento di Fisica, Universitá di Roma Tor Vergata, Via della Ricerca Scientifica 1, I-00133 Rome, Italy and \\ INFN, Laboratori Nazionali di Frascati, Via E. Fermi 40, 00044 Frascati, Italy \\ Stefano Bellucci \\ INFN, Laboratori Nazionali di Frascati, Via E. Fermi 40, 00044 Frascati, Italy \\ (Received 30 September 2012; revised manuscript received 1 January 2013; published 10 January 2013)
}

\begin{abstract}
We consider a quantum ring laterally connected to open one-dimensional leads described within the Luttinger liquid (LL) model and subject to a time-dependent driving magnetic field $\phi(t)$. The closed loop is obtained by connecting two points of the LL by a weak link, which is treated exactly to lowest order in perturbation theory. Analytical results for the current across the weak link, as well as in the external circuit and inside the loop, are obtained. We pay special attention to the case of smooth magnetic pulses such that the response of the system is approximately adiabatic. We find that the response to the flux $\phi$ is linear only if $\phi$ is a small fraction of a flux quantum $\phi_{0}$. By inserting whole quanta, a current goes in the external wires as two bunches, separated by a time proportional to the length of the loop, and a net pumped current in the external wires is produced. This kind of pumping is consistent with the predictions of the corresponding tight-binding model in the uncorrelated limit, but is not destroyed by electron-electron interactions.
\end{abstract}

DOI: 10.1103/PhysRevB.87.035412

PACS number(s): 05.60.Gg, 73.63.Rt

\section{INTRODUCTION}

The magnetic properties of many kinds of nanoscopic devices are attracting increasing attention due to the potential applications in several fields such as spintronics, ${ }^{1}$ optoelectronics, ${ }^{2}$ quantum pumping, and quantum information processing. ${ }^{3}$ A large amount of experimental results have been obtained since the first small quantum rings were fabricated by self-assembled growth of InAs on $\mathrm{GaAs},{ }^{4,5}$ and a complete description of all contributions goes beyond the scope of a single paper. In these systems, the nontrivial topology and the reduced dimensionality are at the origin of a variety of fascinating phenomena such as the Aharonov-Bohm effect, ${ }^{6}$ persistent currents, ${ }^{7}$ Coulomb blockade ${ }^{8}$ and Kondo effect. ${ }^{9}$

From the theoretical side, the general subject of pumping phenomena in mesoscopic or ballistic conductors has been already addressed by several authors. ${ }^{10-15}$ While it is clear by now that pumping is not per se an interaction effect, the inclusion of electron-electron interactions in this problem results in the breakdown of the Fermi liquid, and leads to the formation of the so-called Luttinger liquid (LL). ${ }^{16}$ There is already a considerable literature on the subject of pumping in a LL. ${ }^{17-19}$ Finite length effects in the pumping properties of a LL excited by weak oscillating barriers have been shown ${ }^{20}$ to be negligible in the absence of interactions but to grow large for strong repulsion.

Many properties of the quantum rings can be explained within one-dimensional (1D) models in presence of a closed loop; we here focus on those works in which LL rings coupled to an external circuit have been studied. Symmetric Mach-Zehnder-type geometries have been considered to show striking dependencies of the Aharonov-Bohm amplitude on temperature in the fractional quantum Hall regime, ${ }^{21}$ to study the correlated interference pattern of the electric current, ${ }^{22}$ and to propose an alternative probe to detect the LL state. ${ }^{23}$

It has been stressed lately ${ }^{24}$ that laterally connected rings have unique properties. By laterally connected we mean that the external circuit is tangential to the ring. With this maximally asymmetric connection, a current in the wires can produce a magnetic moment, which is not obtained by substituting the quantum current in the semiclassical formula. It turns out that the circulating current which produces the magnetic moment is localized and does not shift charge from one lead to the other. In contrast with the classical prediction, the linear response current in the ring is always laminar and produces no magnetic moment. ${ }^{24}$ By reversing the process of creating a ring magnetic dipole by a bias-induced current, it was observed that the failure of the linearity assumption yields to the possibility of having nonadiabatic one-parameter pumping. ${ }^{15}$ In particular, it has been shown that if the ring is threaded by a time-varying magnetic field, an arbitrary amount of charge can be transferred from one side to the other, owing to the above-mentioned nonlinear behavior of the magnetic moment as a function of the bias.

In this paper, we take our cue from earlier theoretical results to extend the theory to encompass new systems with ringlike geometries, including the possibility of weak links and above all allowing for correlation effects. Indeed, a weak link behaves like a barrier for the electrons and it is conceivable that such a strong modification spoils the possibility of pumping current in the outside circuit by this method. In order to deal with the interactions, we consider a one-dimensional LL quantum wire containing a closed loop obtained by a weak link and threaded by a time-dependent magnetic flux. We calculate the current generated in the external circuit and across the weak link by switching on the magnetic field according to different 
protocols. For illustration, we insert the flux as a smooth pulse, which is long enough to be approximately adiabatic. Analytical results for the current across the weak link, as well as for the current in the left and the right arms of the circuit and inside the loop are obtained in a nonperturbative treatment of the interaction by bosonizing the fermion operators. The results of numerical evaluations are presented, aiming at a qualitative understanding of the main features.

The case of smooth magnetic pulses, such that the response of the system is approximately adiabatic, is considered in great detail. We find that the response to the flux is linear only if it is a small fraction of a flux quantum. By inserting whole quanta, the current goes in the external wires as two bunches, separated by a time proportional to the length of the loop, and a net pumped current in the external wires is thus produced. We find also that for weak correlations, this behavior is consistent with the predictions of the corresponding tight-binding model. Electron-electron interactions do not modify the trend unless they are very intense.

The plan of the paper goes as follows. In the next section, we outline the model obtained by introducing a weak link joining two sites of an interacting one-dimensional quantum wire. In Sec. III, we show how to calculate the current generated in the external circuit and across the weak link by switching on a magnetic field. The analysis of the results obtained applying the formalism of the previous section is the object of Sec. IV. Finally, in the last section, we provide a summary of our treatment and draw conclusions.

\section{MODEL}

\section{A. Hamiltonian and current operator}

Keeping in mind the geometry depicted in Fig. 1, we start by an interacting one-dimensional quantum wire described by the Tomonaga-Luttinger Hamiltonian

$$
\begin{aligned}
H_{L}= & -\sum_{\alpha} i \epsilon_{\alpha} v_{F} \int d x \psi_{\alpha}^{\dagger}(x) \partial_{x} \psi_{\alpha}(x) \\
& +\frac{1}{2} \int d x\left\{2 g_{2} \rho_{R}(x) \rho_{L}(x)+g_{4}\left[\rho_{R}^{2}(x)+\rho_{L}^{2}(x)\right]\right\},
\end{aligned}
$$

where $\epsilon_{\alpha}= \pm 1$ for $R$ and $L$ electrons moving with velocity $v_{F}$, $\rho_{R / L} \equiv: \psi_{R / L}^{\dagger} \psi_{R / L}:$ is (in standard notation) the fermionic density operator relative to the Fermi sea, and $g_{2 / 4}$ are the forward scattering couplings corresponding to interchirality and intrachirality interactions, respectively. The closed loop of radius $R$ (see Fig. 1) is formed by bending the wire and by connecting the sites at positions $x=0 \equiv A$ and $x=2 \pi R \equiv B$ with a weak link. The perturbation changes the topology. The Hamiltonian describing the weak link is

$$
H_{\lambda}=\lambda e^{i \varphi(t)} \sum_{\alpha, \beta} \psi_{\alpha}^{\dagger}(A) \psi_{\beta}(B)+\text { H.c. }
$$

where the phase $\varphi(t)$ represents the time-dependent magnetic flux threading the ring.

The aim of this work is to calculate the current generated in the external circuit and across the weak link by switching on the magnetic field. The current operator across the link is
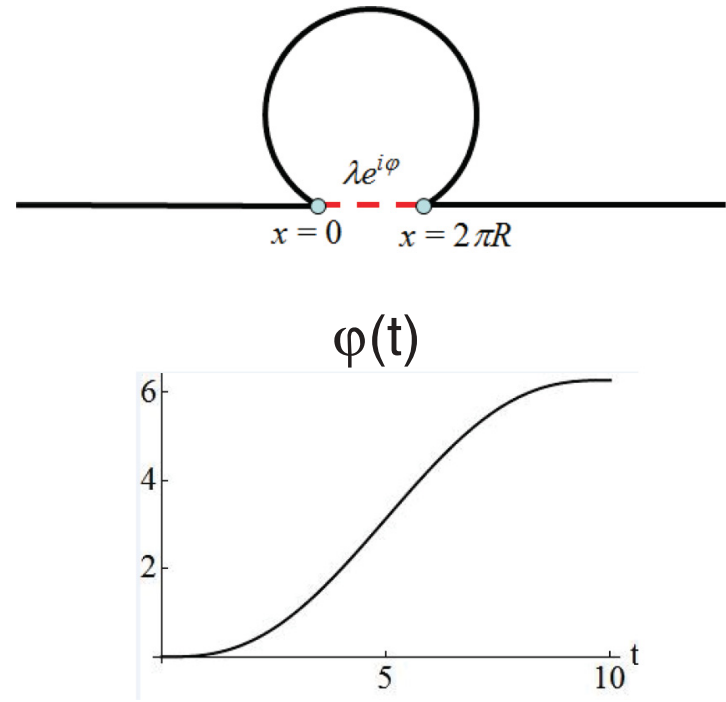

FIG. 1. (Color online) Top: Schematic illustration of the setup geometry. Luttinger liquid wire (solid line) containing a closed loop of radius $R$ generated by a weak link $\lambda$ (dashed line) joining the sites at position 0 and $2 \pi R$. Bottom: The loop is threaded by a time-dependent magnetic flux $\varphi(t)$ given in Eq. (25) that is shown here for $n_{\phi_{0}}=1$. The electromoving force begins smoothly, is maximum for $t=5 t_{0}$, and ends smoothly after a duration $10 t_{0}$.

given by

$J_{\lambda}=i \lambda e^{i \varphi(t)} \sum_{\alpha, \beta} \psi_{\alpha}^{\dagger}(A) \psi_{\beta}(B)+$ H.c. $\equiv \sum_{\alpha, \beta} J_{\alpha \beta}(A, B)$,

while the current operator at a given position $r$ in the wire reads as

$$
J_{r}=v_{F}\left[\rho_{R}(r)-\rho_{L}(r)\right] \equiv \sum_{\alpha} J_{\alpha r} .
$$

For a nonperturbative treatment of the interaction, we bosonize the fermion operators ${ }^{25,26}$

$$
\psi_{\alpha}(x)=\frac{\eta}{\sqrt{2 \pi a}} e^{-2 \sqrt{\pi} i \epsilon_{\alpha} \phi_{\alpha}(x)} e^{i \epsilon_{\alpha} k_{F} x},
$$

where $\eta$ is the anticommuting Klein factor, $k_{F}$ is the Fermi momentum, and $a$ a short-distance cutoff.

\section{B. Mechanical analogy}

To better grasp the physics, the analogy of the timedependent LL (without weak link) with a vibrating string is useful. A transversally vibrating string with fixed ends $X=0$ and $L$ is the simplest continuous dynamical system. ${ }^{27}$ The Lagrangian is

$$
L=\int_{0}^{L}\left[\frac{1}{2} \rho \dot{y}^{2}-\frac{1}{2} T\left(\frac{d y}{d x}\right)^{2}\right],
$$

where $\rho$ is the mass per unit length and $T$ is the tension. The equation of motion is the wave equation $\rho \ddot{y}=T \frac{d^{2} y}{d y^{2}}$ and the wave velocity is $c=\sqrt{\frac{T}{\rho}}$. The canonical momentum is $\Pi=\rho \dot{y}$ and the Hamiltonian reads as

$$
H=\int_{0}^{L}\left[\frac{\Pi(x)^{2}}{2 \rho}+\frac{1}{2} T\left(\frac{d y}{d x}\right)^{2}\right]
$$


To see the analogy, we rewrite the Fermion operators (5) in the form

$$
\psi_{\alpha}(x)=\frac{\eta}{\sqrt{2 \pi a}} e^{i \beta(x)-i \epsilon_{\alpha} \alpha(x)} e^{i \epsilon_{\alpha} k_{F} x},
$$

where

$$
\begin{aligned}
& \alpha(x)=\sqrt{\pi}\left[\phi_{R}(x)+\phi_{L}(x)\right], \\
& \beta(x)=\sqrt{\pi}\left[\phi_{L}(x)-\phi_{R}(x)\right] .
\end{aligned}
$$

Further, we introduce the field

$$
\Pi(x)=\frac{1}{\pi} \frac{d \beta}{d x} .
$$

One can show ${ }^{28}$ that the Luttinger liquid Hamiltonian can be rewritten as

$$
H_{L}=v \int d x\left[\frac{\pi K}{2} \Pi(x)^{2}+\frac{1}{2 \pi K}\left(\frac{d \alpha}{d x}\right)^{2}\right] .
$$

In other terms, the interacting Tomonaga-Luttinger model $H_{L}$ can be written in terms of a transversally vibrating elastic string with density $\rho=\frac{1}{\pi v K}$ and tension $T=\frac{v}{\pi K}$; the wave velocity is $c=v$. The amplitude of vibration is proportional to the sum of the amplitudes of left and right movers. We stress that the string vibrations correspond in the TomonagaLuttinger liquid to density excitations with no single-electron character.

This prompts a mechanical interpretation of the results of the thought experiment. The flux excites the ring and plucks the string. The vibration amplitude of the string is related to the field $\alpha$ and to the density fluctuation of the LL. Indeed, it is well known ${ }^{28}$ that

$$
\frac{d \alpha}{d x}=-\pi\left[\rho(x)-\rho_{0}\right] .
$$

On the other hand, using the continuity equation, one obtains

$$
j(x)=v K \Pi(x) .
$$

Therefore, the current is not related to the vibration amplitude, but to the conjugate variable. We must distinguish the direction of propagation of the disturbance from the direction of the movers: the wave carrying an excess of right movers can propagate to the left.

\section{FORMALISM}

In order to work out the solution, we go back to the formalism of Sec. IA. In the following, we calculate the time-dependent average of $J_{\lambda}$ and $J_{r}$ to the lowest order in $H_{\lambda}$, namely,

$$
\begin{aligned}
j_{\lambda}(t)=i\left\langle\Psi_{0}\left|\int_{0}^{t} d s\left[H_{\lambda}(s), J_{\lambda}(t)\right]\right| \Psi_{0}\right\rangle, \\
j_{r}(t)=\left\langle\Psi_{0}\right|\left\{-\int_{0}^{t} d t_{1} \int_{0}^{t_{1}} d t_{2}\left[H_{\lambda}\left(t_{2}\right) H_{\lambda}\left(t_{1}\right) J_{r}(t)\right.\right. \\
\left.+J_{r}(t) H_{\lambda}\left(t_{1}\right) H_{\lambda}\left(t_{2}\right)\right] \\
\left.+\int_{0}^{t} \int_{0}^{t} d t_{1} d t_{2} H_{\lambda}\left(t_{1}\right) J_{r}(t) H_{\lambda}\left(t_{2}\right)\right\}\left|\Psi_{0}\right\rangle,
\end{aligned}
$$

where $\left|\Psi_{0}\right\rangle$ is the ground state of $H_{L}$ and operators are in the Heisenberg representation with respect to $H_{L}$. The above time-dependent averages have the following physical meaning: since they are evaluated over the uncontacted ground state of $H_{L}$, their temporal evolution occurs as the weak link was switched on at time $t=0$. This is the so-called partitioned approach ${ }^{29-31}$ to the time-dependent transport. Since the function $\varphi(t)$ describing the time-varying piercing magnetic flux is completely arbitrary, we can first evolve the system with $\varphi(t)=0$ under the only effect of closing the ring and let the system relax. After relaxation, say at time $t_{0}$, we switch on the magnetic flux and study the dynamics from the transient to the steady state. This procedure simulates with high accuracy the so-called partition-free scheme, ${ }^{29,30}$ as demonstrated in Refs. 31-33. At any rate, since we insert the flux slowly, the system has time to relax and all that happens after a time much longer than $t_{0}$ can be understood as taking place in a partition-free scheme. In the next sections, we use the bosonization method to calculate explicitly $j_{\lambda}(t)$ and $j_{r}(t)$.

\section{A. Current across the weak link}

The great advantage of the bosonization technique is that the interacting ground state $\left|\Psi_{0}\right\rangle$ appearing in Eqs. (14) and (15) is nothing but the vacuum of the boson operators $b_{\alpha q}$ entering in the mode expansion

$$
\phi_{\alpha}(x)=i \epsilon_{\alpha} \sum_{q>0} \frac{e^{-a q / 2}}{\sqrt{2 \mathcal{L} q}}\left[C_{+} b_{\alpha q}^{\dagger}-C_{-} b_{\bar{\alpha} q}\right] e^{-i \epsilon_{\alpha} q}+\text { H.c. },
$$

where $\mathcal{L}$ is the length of the wire. ${ }^{34}$ The coefficients $C_{ \pm}$carry all the information about the electron-electron interaction and are given by

$$
C_{ \pm}=\frac{1 \pm K}{2 \sqrt{K}}
$$

with

$$
K=\sqrt{\frac{2 \pi v_{F}+g_{4}-g_{2}}{2 \pi v_{F}+g_{4}+g_{2}}}
$$

the Luttinger liquid (LL) parameter which measures the interaction strength. ${ }^{16}$ Note that $0<K \leqslant 1$ for repulsive interactions; $K=1$ corresponds to the noninteracting case, while small values of $K$ indicate a strongly correlated regime. It is worth recalling that in the Heisenberg representation of Eqs. (14) and (15), the explicit time dependence of the operators is accounted for by using $b_{\alpha q}(t)=e^{-i q v t} b_{\alpha q}$, with

$$
v=\frac{\sqrt{\left(2 \pi v_{F}+g_{4}\right)^{2}-g_{2}^{2}}}{2 \pi}
$$

the velocity of the interacting quasiparticles.

It is straightforward to verify that the current across the link can be rewritten as $j_{\lambda}(t)=\sum_{\alpha \beta} j_{\alpha \beta}(A, B, t)$, where $j_{\alpha \beta}(A, B, t)$ is the average obtained by replacing the operator $J_{\lambda}$ in Eq. (14) with the operator $J_{\alpha \beta}(A, B)$. In order to evaluate the different contributions $j_{\alpha \beta}(A, B, t)$, it is convenient to express 
them in terms of the fundamental correlator

$g_{\alpha \beta \gamma \delta}(x y z w, s t)=\left\langle\Psi_{0}\left|\psi_{\alpha}^{\dagger}(x, s) \psi_{\beta}(y, s) \psi_{\gamma}^{\dagger}(z, t) \psi_{\delta}(w, t)\right| \Psi_{0}\right\rangle$.

The explicit evaluation of $g$ requires some lengthy bosonization algebra, which we here omit. For instance, the current $j_{R R}(A, B, t)$ reads as

$$
\begin{aligned}
j_{R R}(A, B, t)= & -2 \lambda^{2} \int_{0}^{t} d s \operatorname{Re}\left\{g_{R R R R}(A B A B, s t) e^{i[\varphi(s)+\varphi(t)]}\right. \\
& +g_{R R R R}(B A A B, s t) e^{i[-\varphi(s)+\varphi(t)]} \\
& +g_{L L R R}(A B A B, s t) e^{i[\varphi(s)+\varphi(t)]} \\
& +g_{L L R R}(B A A B, s t) e^{i[-\varphi(s)+\varphi(t)]} \\
& -g_{R R R R}(A B B A, s t) e^{i[\varphi(s)-\varphi(t)]} \\
& -g_{R R R R}(B A B A, s t) e^{i[-\varphi(s)-\varphi(t)]} \\
& -g_{L L R R}(A B B A, s t) e^{i[\varphi(s)-\varphi(t)]} \\
& \left.-g_{L L R R}(B A B A, s t) e^{i[-\varphi(s)-\varphi(t)]}\right\},
\end{aligned}
$$

while the current $j_{R L}(A, B, t)$ is

$$
\begin{aligned}
j_{R L}(A, B, t)= & -2 \lambda^{2} \int_{0}^{t} d s \operatorname{Re}\left\{g_{R L R L}(A B A B, s t) e^{i[\varphi(s)+\varphi(t)]}\right. \\
& +g_{R L R L}(B A A B, s t) e^{i[-\varphi(s)+\varphi(t)]} \\
& +g_{L R R L}(A B A B, s t) e^{i[\varphi(s)+\varphi(t)]} \\
& +g_{L R R L}(B A A B, s t) e^{i[-\varphi(s)+\varphi(t)]} \\
& -g_{R L R L}(A B B A, s t) e^{i[\varphi(s)-\varphi(t)]} \\
& -g_{R L R L}(B A B A, s t) e^{i[-\varphi(s)-\varphi(t)]} \\
& -g_{L R R L}(A B B A, s t) e^{i[\varphi(s)-\varphi(t)]} \\
& \left.-g_{L R L R}(B A B A, s t) e^{i[-\varphi(s)-\varphi(t)]}\right\} .
\end{aligned}
$$

The contributions $j_{L L}(A, B, t)$ and $j_{L R}(A, B, t)$ are obtained by exchanging $L \leftrightarrow R$ in the above expressions.

\section{B. Current in the wires}

The evaluation of the current at a given point of the wire is more involved because there are three different terms in Eq. (15), and because each correlator involves three oneparticle operators. Let us denote by $S, T$, and $V$ the averages in three lines of Eq. (15), and let us evaluate each contribution separately.

We first observe that in the bosonization language, the chiral current operator reads as $J_{\alpha r}=\epsilon_{\alpha} v_{F} \partial_{r} \phi_{\alpha}(r) / \sqrt{\pi}$. The contributions $S$ and $T$ can be expressed in terms of the fundamental correlator

$$
\begin{aligned}
G_{\alpha \beta \gamma \delta}^{\mu}\left(x y z w r, t_{1} t_{2} t\right)= & \left\langle\Psi_{0}\right| \psi_{\alpha}^{\dagger}\left(x, t_{1}\right) \psi_{\beta}\left(y, t_{1}\right) \psi_{\gamma}^{\dagger}\left(w, t_{2}\right) \\
& \times \psi_{\delta}\left(w, t_{2}\right) \partial_{r} \phi_{\mu}(r)\left|\Psi_{0}\right\rangle,
\end{aligned}
$$

the explicit calculation of which is cumbersome and is not shown here (the same also holds for the correlator $F$, see following). Thus, the contribution $S$ reads as

$$
\begin{aligned}
S(r, t)= & -\frac{\lambda^{2} v_{F}}{\sqrt{\pi}} \sum_{\alpha \beta \mu} \epsilon_{\mu} \int_{0}^{t} d t_{1} \int_{0}^{t_{1}} d t_{2} \\
& \times\left\{G_{\alpha \alpha \beta \beta}^{\mu}\left(A B A B r, t_{1} t_{2} t\right) e^{i\left[\varphi\left(t_{1}\right)+\varphi\left(t_{2}\right)\right]}\right. \\
& +G_{\alpha \alpha \beta \beta}^{\mu}\left(A B B A r, t_{1} t_{2} t\right) e^{i\left[\varphi\left(t_{1}\right)-\varphi\left(t_{2}\right)\right]}
\end{aligned}
$$

$$
\begin{aligned}
& +G_{\alpha \alpha \beta \beta}^{\mu}\left(B A A B r, t_{1} t_{2} t\right) e^{i\left[-\varphi\left(t_{1}\right)+\varphi\left(t_{2}\right)\right]} \\
& +G_{\alpha \alpha \beta \beta}^{\mu}\left(B A B A r, t_{1} t_{2} t\right) e^{i\left[-\varphi\left(t_{1}\right)-\varphi\left(t_{2}\right)\right]} \\
& +\delta_{\alpha, \beta} G_{\alpha \bar{\alpha} \bar{\beta} \beta}^{\mu}\left(A B A B r, t_{1} t_{2} t\right) e^{i\left[\varphi\left(t_{1}\right)+\varphi\left(t_{2}\right)\right]} \\
& +\delta_{\alpha, \beta} G_{\alpha \bar{\alpha} \bar{\beta} \beta}^{\mu}\left(A B B A r, t_{1} t_{2} t\right) e^{i\left[\varphi\left(t_{1}\right)-\varphi\left(t_{2}\right)\right]} \\
& +\delta_{\alpha, \beta} G_{\alpha \bar{\alpha} \bar{\beta} \beta}^{\mu}\left(B A A B r, t_{1} t_{2} t\right) e^{i\left[-\varphi\left(t_{1}\right)+\varphi\left(t_{2}\right)\right]} \\
& \left.+\delta_{\alpha, \beta} G_{\alpha \bar{\alpha} \bar{\beta} \beta}^{\mu}\left(B A B A r, t_{1} t_{2} t\right) e^{i\left[-\varphi\left(t_{1}\right)-\varphi\left(t_{2}\right)\right]}\right\},
\end{aligned}
$$

whereas $T$ is simply obtained as $T(r, t)=S^{*}(r, t)$.

The contribution $V$ instead involves the different correlator

$$
\begin{aligned}
F_{\alpha \beta \gamma \delta}^{\mu}\left(x y z w r, t_{1} t_{2} t\right)= & \left\langle\Psi_{0}\right| \psi_{\alpha}^{\dagger}\left(x, t_{1}\right) \psi_{\beta}\left(y, t_{1}\right) \partial_{r} \phi_{\mu}(r) \\
& \times \psi_{\gamma}^{\dagger}\left(w, t_{2}\right) \psi_{\delta}\left(w, t_{2}\right)\left|\Psi_{0}\right\rangle,
\end{aligned}
$$

in terms of which we have

$$
\begin{aligned}
T(r, s)= & \frac{\lambda^{2} v_{F}}{\sqrt{\pi}} \sum_{\alpha \beta \mu} \epsilon_{\mu} \int_{0}^{t} \int_{0}^{t} d t_{1} d t_{2} \\
& \times\left\{F_{\alpha \alpha \beta \beta}^{\mu}\left(A B A B r, t_{1} t_{2} t\right) e^{i\left[\varphi\left(t_{1}\right)+\varphi\left(t_{2}\right)\right]}\right. \\
& +F_{\alpha \alpha \beta \beta}^{\mu}\left(A B B A r, t_{1} t_{2} t\right) e^{i\left[\varphi\left(t_{1}\right)-\varphi\left(t_{2}\right)\right]} \\
& +F_{\alpha \alpha \beta \beta}^{\mu}\left(B A A B r, t_{1} t_{2} t\right) e^{i\left[-\varphi\left(t_{1}\right)+\varphi\left(t_{2}\right)\right]} \\
& +F_{\alpha \alpha \beta \beta}^{\mu}\left(B A B A r, t_{1} t_{2} t\right) e^{i\left[-\varphi\left(t_{1}\right)-\varphi\left(t_{2}\right)\right]} \\
& +\delta_{\alpha, \beta} F_{\alpha \bar{\alpha} \bar{\beta} \beta}^{\mu}\left(A B A B r, t_{1} t_{2} t\right) e^{i\left[\varphi\left(t_{1}\right)+\varphi\left(t_{2}\right)\right]} \\
& +\delta_{\alpha, \beta} F_{\alpha \bar{\alpha} \bar{\beta} \beta}^{\mu}\left(A B B A r, t_{1} t_{2} t\right) e^{i\left[\varphi\left(t_{1}\right)-\varphi\left(t_{2}\right)\right]} \\
& +\delta_{\alpha, \beta} F_{\alpha \bar{\alpha} \bar{\beta} \beta}^{\mu}\left(B A A B r, t_{1} t_{2} t\right) e^{i\left[-\varphi\left(t_{1}\right)+\varphi\left(t_{2}\right)\right]} \\
& \left.+\delta_{\alpha, \beta} F_{\alpha \bar{\alpha} \bar{\beta} \beta}^{\mu}\left(B A B A r, t_{1} t_{2} t\right) e^{i\left[-\varphi\left(t_{1}\right)-\varphi\left(t_{2}\right)\right]}\right\} .
\end{aligned}
$$

Collecting all contributions together, we finally have $j_{r}(t)=$ $S(r, t)+T(r, t)+V(r, t)$. We recall that the above functions allow us to evaluate the current in the left and right arms of the circuit, as well as inside the loop. To this end, we have to set $r<0, r>2 \pi R$, and $0<r<2 \pi R$, respectively (see Fig. 1).

\section{Switching protocol}

The numerical results presented in the next section are obtained by choosing the following smooth protocol for switching $n_{\phi_{0}}$ flux quanta (see Fig. 1):

$$
\frac{\varphi(t)}{n_{\phi_{0}}}=\left\{\begin{array}{l}
0, \quad t<0 \\
2 \pi\left[10\left(\frac{t}{t_{d}}\right)^{3}-15\left(\frac{t}{t_{d}}\right)^{4}+6\left(\frac{t}{t_{d}}\right)^{5}\right], \quad t<t_{d} \\
2 \pi, \quad t>t_{d}
\end{array}\right.
$$

where $t_{d}$ is the duration of the switching. The LL has a characteristic time $t_{0}$ (see below), and in order to avoid overheating the sample we opt for $t_{d}=10 t_{0}$; the switching protocol is smooth and slow, and we may expect an approximately adiabatic response of the system.

\section{RESULTS AND DISCUSSION}

\section{A. Propagation of the pulse}

In this section and in the rest of the paper, ${ }^{35}$ lengths are expressed in units of $x_{0}=10 a$, time in units of $t_{0}=10 a / v_{F}$, 
current in units of $j_{0}=v_{F} /(10 a)$, the Fermi momentum in units of $k_{0}=1 /(10 a)$, and the renormalized velocity $v$ in units of $v_{F}$. For simplicity, in the numerical results we have assumed $v=v_{F}$. The ring circumference is $C \equiv 2 \pi R=20$ and the Fermi momentum is $k_{F}=1$, but the results depend weakly on the latter.

First of all we note that even in the presence of the weak link it makes sense to speak of left and right movers (referring to the limit when the link disappears). In the ring, a clockwise current means an excess of right movers. By continuity, this implies that in the weak link a clockwise current means $j<0$.

In Fig. 2, we show typical results obtained by evaluating numerically the functions derived above with $n_{\phi_{0}}=2$. The model is definitely interacting, with LL parameter $K=0.6$. In the top left panel, we see the behavior of the current right at the weak link. As dictated by the sign of the magnetic field, the current goes clockwise with a spike-shaped transient behavior. The shape and width of the peak with a minimum about $t=5 t_{0}$ reflect the behavior of $-\frac{d \varphi(t)}{d t}$. When this response to the electromotive force (emf) is over the current vanishes until at time $t=30$, at which some excitation is again seen. This excitation has traveled with unit speed $v=1$ as expected from the string analogy and returns to the weak link at time $C / v$, i.e., after completing a tour of the ring. The top-right panel of Fig. 2 shows the current calculated at $x=5$ in the right wire. Nothing is seen initially for it takes $5 t_{0}$ for the signal to arrive there from a source which is apparently located at the weak link. Then, the negative spike is seen to arrive. This is followed after a $20 t_{0}=C / v$ delay by a roughly opposite signal. In Ref. 15 , a flux threading a laterally connected tight-binding
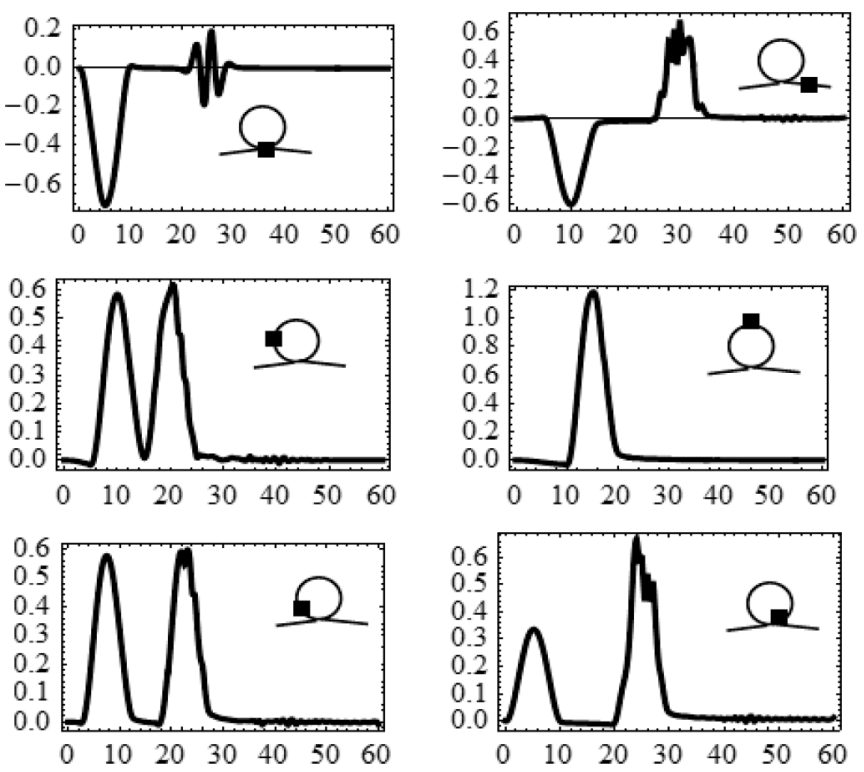

FIG. 2. Current versus time for $n_{\phi_{0}}=2$ at various positions in the system. The emf is nonzero only up to $t=10$. A rather strong repulsive electron-electron interaction is assumed $(K=0.6)$. The dark squares indicate the position where the current $j(t)$ is measured in each panel. Top left: at the weak link. Top right: in the right wire at $x=5 x_{0}$. The same $j(t)$ holds in the left wire at $x=-5 x_{0}$ and there is full left-right symmetry. Middle left: at $\frac{1}{4}$ of the ring; the same result is obtained at $\frac{3}{4}$ of the ring. Middle right: in the middle of ring. Bottom left: $\frac{1}{8}$ of the ring. Bottom right: at $99 \%$ of ring. ring was shown to pump current in the external circuit. Here, the pumped current is largely canceled by the second spike. The difference is not due to the presence of interactions; the main reason is that in Ref. 15 a perfectly regular ring was considered, while here the weak link suppresses the circulating current. However, in practice the pumping is still possible. One can delay at will the opposite signal by making $C$ larger and larger, and at the limit one gets charge pumping. One can also get the pumping by introducing in the circuit a nonlinear rectifying element. Remarkably, in the left wire at the symmetric location $x=-5 x_{0}$, the current $j(t)$ is the same (we stress, the same, not opposite). The effect of the flux in the ring is that of producing the same current in both wires, with a burst of left current followed by a roughly opposite burst after a time $C / v$.

Next, let us have a look on what happens inside the ring. There, an excess of right movers $(j>0)$ is seen. The wave propagates symmetrically towards the right and the left in the external circuit. As noted above, the direction of propagation of the disturbance does not tell the direction of the movers: the wave carrying an excess of right movers can propagate to the left.

In the middle left panel, the current is evaluated at a quarter of the ring, where an excess of right movers arrives in two waves. The first maximum arrives at $t=10=5 t_{0}+\frac{C}{4 v}$. Since $5 t_{0}$ is the time it takes to the emf to reach the maximum, it is clear that these right movers started from the weak link clockwise. The second wave has a maximum at $t=5 t_{0}+\frac{3 C}{4 v}$. They are still right movers. However, they are also roundabout movers that arrived the other way around. In the same way, we understand how an identical pattern is obtained at $\frac{3}{4}$ of the ring (not shown). In the middle right panel, the current is calculated at the center of the ring, where the two waves arrive together from opposite sides. Now, it is clear that the burst of right movers that we see arriving at $\frac{1}{4}$ ring at $t=10$ at half ring at $t=15 t_{0}$ and at $\frac{3}{4}$ ring $t=20 t_{0}$ are the same that at $t=30 t_{0}$ arrive at $x=5 x_{0}$ and the symmetric burst at $x=-5 x_{0}$ is formed by the counterpropagating right movers that we observed in the ring. The bottom-left panel shows that at $\frac{1}{8}$ of the ring, the story is the same as at $\frac{1}{4}$ ring, but everything happens earlier. The last picture of Fig. 2 shows that at the end of the ring the current is not the same as in the first picture because some of it ends into the external circuit.

The scheme of the complete evolution is illustrated in Fig. 3 aiming at an overall understanding of the phenomena. From the whole of Fig. 2 one can make up a clear picture. The first negative pulse in Fig. 2 top right $\left(x=5 x_{0}\right)$ comes directly from the right end of weak link, and is represented by the black arrow in Fig. 3 top pointing to left. The second positive pulse in the right wire comes from arrow in the ring arising from the left side of the weak link and arrives after a tour of the ring still pointing to the right (Fig. 3 bottom). Similarly, the second positive pulse in the left wire arises from the arrow close to the right end of the weak link in Fig. 3 top panel.

\section{B. Flux dependence}

We now address the question of how the current response depends on the total flux. In Fig. 4, we show that the 


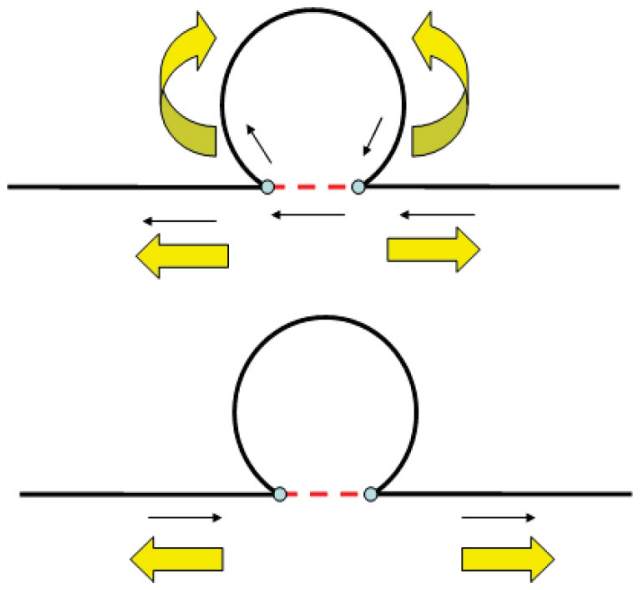

FIG. 3. (Color online) Pattern of the currents (black arrows) and of the propagation of the disturbance (block arrows). Upper: the scheme during or shortly after the flux penetration. Lower: the longtime propagation of the right movers in the wires. The black arrows that in the upper panel are in the ring have reached the wires without crossing the weak link. Here, we have taken $K=0.6$.

dependence of the current on $n_{\phi_{0}}$ is linear for $n_{\phi_{0}} \ll 1$, although for $n_{\phi_{0}}=0.2$ some deviations can already be noticed, particularly in the strong suppression of a long-time tail which follows the initial response to the emf and the feature at $t=C / v_{F}$ arising from the excitation that runs through all the ring. At higher $n_{\phi_{0}}$, the response is less than proportional to the flux and the general shape of the initial response during the emf pulse becomes stubby, while the roundabout structure becomes much more complex.

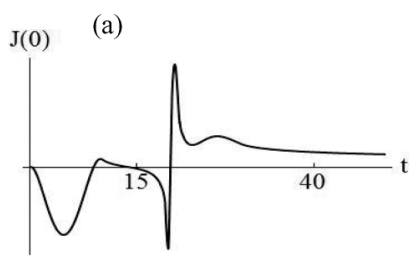

(c)

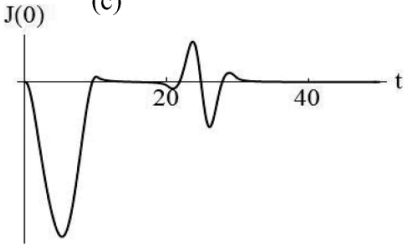

(e)
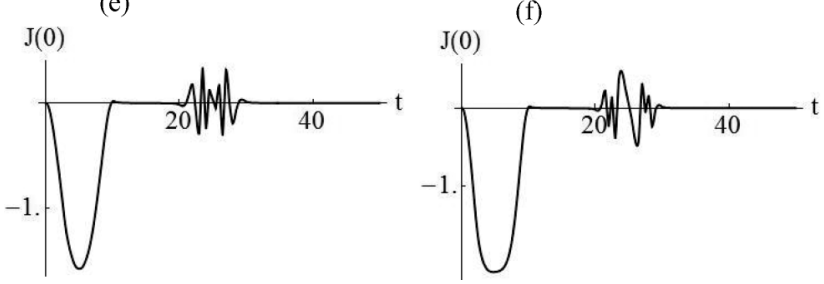

FIG. 4. Current at the weak link versus time for various $n_{\phi_{0}}$. (a) $n_{\phi_{0}}=0.1$; (b) $n_{\phi_{0}}=0.2$; (c) $n_{\phi_{0}}=1$; (d) $n_{\phi_{0}}=2$; (e) $n_{\phi_{0}}=5$; (f) $n_{\phi_{0}}=10$. Here, we have taken $K=0.6$.
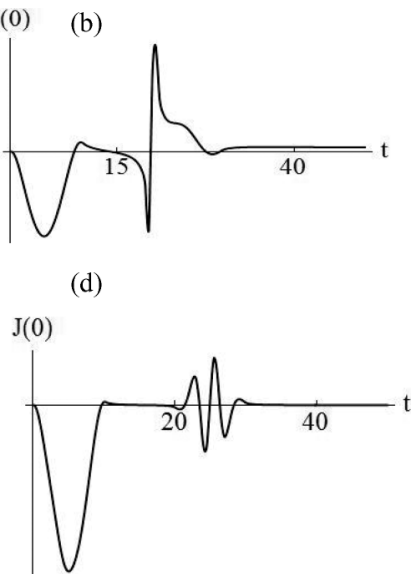

(d)
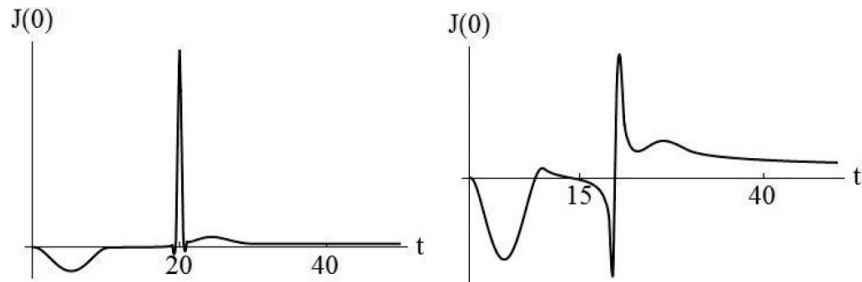

FIG. 5. Current at the weak link versus time as obtained using the parameters given in the text, with $n_{\phi_{0}}=1$. Left: $K=1$. Right: $K=0.6$.

\section{Comparison with the noninteracting case}

In Fig. 5, the current at the weak link for $K=1$ noninteracting case (left) is compared to the $K=0.6$ response (right) (moderately strong repulsive interaction). In both cases, $n_{\phi_{0}}=0.1$. The noninteracting system gives an almost three times stronger response up to $t=t_{d}$ when the phase depends on time, and a strong sharp spike at $t=20$ when the carriers arrive after covering the ring. In the interacting case, the response at $t=20$ is wider and more complex with a change of sign and a long tail. The different behavior can be rationalized since the system must take energy from the magnetic field and one knows that the excited spectrum of the system depends on the interaction parameter $K$.

In order to convey an impression of the effects of the interaction, in Fig. 6 we show the evolution with $n_{\phi_{0}}=1$ of the current at $x=5 x_{0}$ along the right wire at different $K$ values
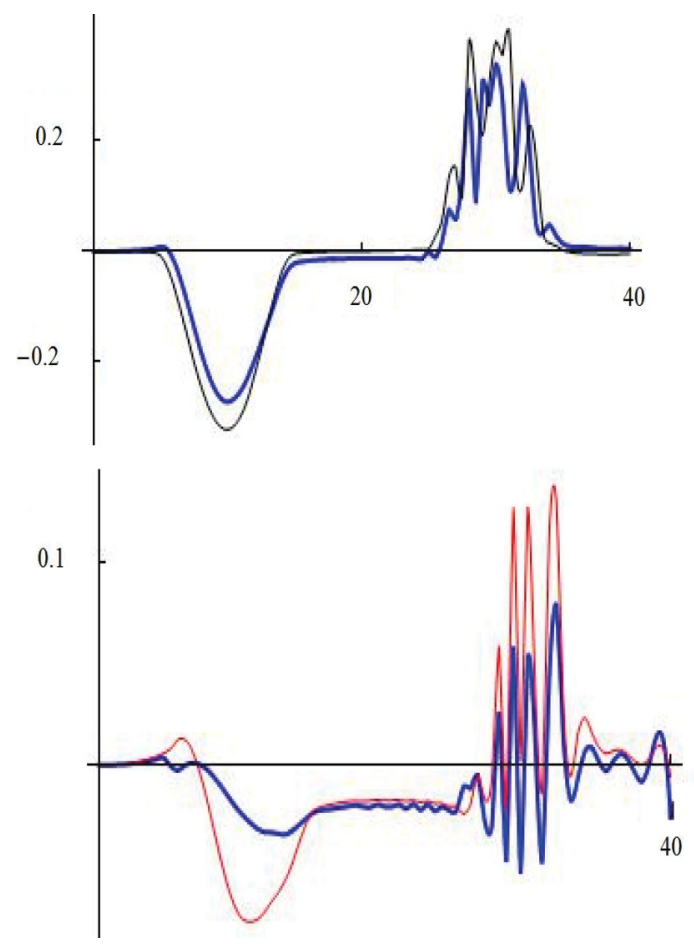

FIG. 6. (Color online) Current at $x=5 x_{0}$ in the right wire versus time as obtained using the parameters given in the text. Top panel (note the different scale): heavy line: $K=0.6$; light line: $K=1$. Bottom panel: heavy line: $K=0.3$; light line: $K=0.4$. The current at $x=-5 x_{0}$ in the left wire is the same. 
(or at at $x=-5 x_{0}$ along the left wire). In the top panel, we compare the noninteracting case $(K=1)$ with a repulsive case $(K=0.6)$. The first spike of current reaches the observation point with unit speed from the weak link and is followed by the now familiar roundabout spike. The (approximately adiabatic) uptake of one flux quantum ensures that the ring returns close to the ground state after the disturbance. Although the difference between the two curves is evident, we may say that the interactions entail a modest decrease of the current while the electromoving force is on; one can notice some change in the structure of the peak around $t=20$. No significant excitation remains after this time.

In the bottom panel, we compare $(K=0.4)$ with $(K=$ 0.3 ). Now, the repulsion is quite strong and the decrease in $K$ progressively reduces both spikes of current. In terms of the mechanical analogy of Sec. II B, we find that increasing the tension and the mass density of the string, the amplitude of the oscillation decreases. Moreover, the system has memory and the first spike at $t=\frac{5 x_{0}}{v}$ has a tail that lasts until the roundabout current arrives at time $\frac{2 \pi R+5 x_{0}}{v}$. Nevertheless, there is pumping until then, and this arrival can be delayed by increasing $R$ or avoided at all by inserting a switch. The most significant conclusion is that the effect of the interactions does not seriously hamper the behavior predicted in Ref. 15. For very strong interactions $(K<0.5)$, the results of the present approach can not perhaps be taken too literally because of known technical problems, ${ }^{17,19}$ which are beyond the scope of this paper. At any rate, the qualitative prediction is still that a one-parameter pumping is achievable as explained above.

\section{Comparison with the tight-binding model}

The LL model differs so much from the tight-binding description that even in the $K=1$ case one can not expect any quantitative agreement. However, since both theories aim to describe the same system, it would be worrying if no analogy existed at all. Therefore, we considered a tight-binding model with a $N_{a}$ atom ring. All the sides of the polygon correspond to a hopping integral $\tau=1$ except one with a weak link $\tau / 100$ connected to 200 atom wires. In the wires, we take $\tau=1$ and we measure times in $1 / \tau$ units. The finite length of the wires causes a rebound current after a long enough time so that we can safely forget about it. ${ }^{36}$ The band is taken as half-filled. For inserting the flux in the ring, the same protocol as in Eq. (25) is employed. The results are shown in Fig. 7 and can be compared to Fig. 4(c). There are obvious differences. The pattern is complicated by a single discrete excitation frequency of order 2. The response during the emf is positive but changes sign while the phase is still increasing. The analogies are a burst of current during the emf and a feature occurring with a delay $\sim 20$ corresponding to the times that it takes the carriers to go around the ring. Moreover, there is a net pumping of current as discussed in Ref. 15 despite the weak link. This feature remains true in the Luttinger model too. We then see that tight-binding and LL approaches describe basically the same physics, although the two descriptions imply different excitations and different state densities.

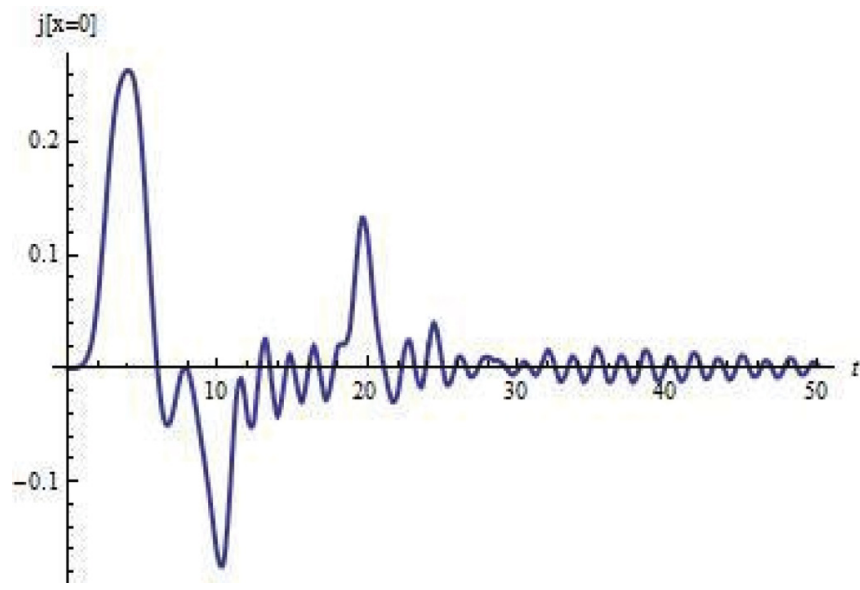

FIG. 7. (Color online) Current at the weak link versus time as obtained using the tight-binding model with $n_{\phi_{0}}=1$.

\section{SUMMARY AND CONCLUSIONS}

We have considered an interacting quantum ring where a time-dependent magnetic flux creates a magnetic moment and through it excites a current in an external circuit. In order to excite a current in a ballistic circuit by threading an external magnetic field through a ring, the geometry must not be symmetric. In this paper, the laterally bonded ring is realized by twisting an interacting Luttinger liquid wire. The presence of a weak link allows us to solve the problem in second-order perturbation theory. The weak link also makes a major physical difference with respect to a perfect ring because both ends act as sources of excitations of the elastic string analog of the Luttinger liquid. In the ring and in the wires, two pulses of current are observed, coming from both ends of the weak link; the roundabout current coming from the far end arrives with a delay proportional to the ring circumference. While the elastic string description is specific of the Luttinger liquid, at a more qualitative level, something similar occurs in the tight-binding model as well, where the pulse coming from the far end of the weak link is also predicted.

For illustration we have chosen to insert the flux as a smooth pulse, which is long enough to be approximately adiabatic. Indeed, when the inserted flux is an integer number of flux quanta, the ring remains close to the ground state and nothing happens in the wires when the corresponding current pulses have passed. On the other hand, when a fraction of a flux quantum is introduced, the current pulses have a tail due to the persistent excitations in the ring. The response to the magnetic flux is linear when the number $n_{\phi_{0}}$ of inserted flux quanta is much less than unity; otherwise, the response grows less than linearly and the shape of the current as a function of time is different at one and two fluxons. There is a general but gradual decrease of the current with increasing interactions.

We have suggested elsewhere ${ }^{15}$ by a tight-binding model that in the ballistic regime one can pump current in a circuit by exciting a ring by an external time-dependent magnetic field. The main result of this paper is that the inclusion of electron-electron interactions and of the weak link modifies many details but does not hamper this interesting behavior. 
${ }^{1}$ S. Souma and B. K. Nikolic, Phys. Rev. B 70, 195346 (2004).

${ }^{2}$ Z. Barticevic, M. Pacheco, and A. Latge, Phys. Rev. B 62, 6963 (2000).

${ }^{3}$ P. Foldi, O. Kalman, M. G. Benedict, and F. M. Peeters, Nano Lett. 8, 2556 (2008); O. Kalman, P. Foldi, M. G. Benedict, and F. M. Peeters, Phys. Rev. B 78, 125306 (2008).

${ }^{4}$ R. J. Warburton, C. Schaflein, D. Haft, F. Bickel, A. Lorke, K. Karrai, J. M. Garcia, W. Schoenfeld, and P. M. Petroff, Nature (London) 405, 926 (2000).

${ }^{5}$ A. Lorke, R. J. Luyken, A. O. Govorov, J. P. Kotthaus, J. M. Garcia, and P. M. Petroff, Phys. Rev. Lett. 84, 2223 (2000).

${ }^{6}$ Y. Aharonov and D. Bohm, Phys. Rev. 115, 485 (1959).

${ }^{7}$ M. Buttiker, Y. Imry, and R. Landauer, Phys. Lett. A 96, 365 (1983).

${ }^{8}$ A. Fuhrer, S. Lusher, T. Ihn, T. Heinzel, K. Ensslin, W. Wegscheider, and M. Bichler, Nature (London) 413, 822 (2001).

${ }^{9}$ U. F. Keyser, C. Fuhner, S. Borck, R. J. Haug, M. Bichler, G. Abstreiter, and W. Wegscheider, Phys. Rev. Lett. 90, 196601 (2003).

${ }^{10}$ M. Moskalets and M. Buttiker, Phys. Rev. B 66, 205320 (2002).

${ }^{11}$ M. Moskalets and M. Buttiker, Phys. Rev. B 66, 035306 (2002).

${ }^{12}$ L. Arrachea, Phys. Rev. B 72, 121306 (2005); 72, 249904 (2005).

${ }^{13}$ L. Arrachea, C. Naon, and M. Salvay, Phys. Rev. B 76, 165401 (2007).

${ }^{14}$ R. Citro and F. Romeo, Phys. Rev. B 73, 233304 (2006).

${ }^{15}$ M. Cini and E. Perfetto, Phys. Rev. B 84, 245201 (2011).

${ }^{16}$ F. D. M. Haldane, J. Phys. C: Solid State Phys. 14, 2585 (1981).

${ }^{17}$ A. Agarwal and D. Sen, Phys. Rev. B 76, 035308 (2007).

${ }^{18}$ M. J. Salvay, Phys. Rev. B 79, 235405 (2009).

${ }^{19}$ D. E. Feldman and Yuval Gefen, Phys. Rev. B 67, 115337 (2003).

${ }^{20}$ S. Franchino Viñas, P. Pisani, and M. Salvay, Eur. Phys. J. B 85, 136 (2012).
${ }^{21}$ M. R. Geller and D. Loss, Phys. Rev. B 56, 9692 (1997).

${ }^{22}$ T. Jonckheere, P. Devillard, A. Crepieux, and T. Martin, Phys. Rev. B 72, 201305 (2005).

${ }^{23}$ M. R. Geller, Phys. Rev. Lett. 80, 5393 (1998).

${ }^{24}$ M. Cini, E. Perfetto, and G. Stefanucci, Phys. Rev. B 81, 165202 (2010).

${ }^{25}$ T. Giamarchi, Quantum Physics in One Dimension (Clarendon, Oxford, 2004).

${ }^{26}$ J. Gonzàlez, M. A. Martín-Delgado, G. Sierra, and M. A. H. Vozmediano, Quantum Electron Liquids and High- $T_{c}$ Superconductivity (Springer, Berlin, 1995).

${ }^{27}$ Michael Stone and Paul Goldbarth, Mathematics for Physics: A Guided Tour for Graduate Students (Cambridge University Press, Cambridge, UK, 2009).

${ }^{28}$ H. J. Schulz, G. Cuniberti, and P. Pieri, in Field Theories for LowDimensional Condensed Matter Systems, edited by G. Morandi et al. (Springer, Berlin, 2000).

${ }^{29}$ M. Cini, Phys. Rev. B 22, 5887 (1980).

${ }^{30}$ G. Stefanucci and C. O. Almbladh, Phys. Rev. B 69, 195318 (2004).

${ }^{31}$ E. Perfetto, G. Stefanucci, and M. Cini, Phys. Rev. Lett. 105, 156802 (2010).

${ }^{32}$ P. Myöhänen et al., Europhys. Lett. 84, 67001 (2008).

${ }^{33}$ V. Moldoveanu, H. D. Cornean, and C.-A. Pillet, Phys. Rev. B 84, 075464 (2011)

${ }^{34}$ At the end of the calculation of any boson average, the length $\mathcal{L}$ must be sent to infinity, by remembering that $\frac{2 \pi}{\mathcal{L}} \sum_{q>0} \rightarrow \int_{0}^{\infty} d q$.

${ }^{35}$ In this paper, we use $e=\hbar=1$.

${ }^{36}$ E. Perfetto, G. Stefanucci, and M. Cini, Phys. Rev. B 78, 155301 (2008); 80, 205408 (2009); 82, 035446 (2010); M. Cini, E. Perfetto, C. Ciccarelli, G. Stefanucci, and S. Bellucci, ibid. 80, 125427 (2009). 